\title{
Red de Sensores Inalámbricos de Bajo Consumo Energético en Agricultura Hidropónica
}

\author{
Carlos Cambra ${ }^{1}$, Sandra Sendra ${ }^{1,2}$, José Miguel Jiménez ${ }^{2}$, Jaime Lloret $^{2}$ \\ ${ }^{1}$ Instituto de Investigación para la Gestión Integrada de zonas Costeras. Universitat Politècnica de València, \\ Valencia \\ C/Paraninf, 1. 46730. Grao de Gandia (Valencia) \\ ${ }^{2}$ Departamento de teoria de la señal, telemática y comunicaciones, ETS Ingenierías Informática y de \\ Telecomunicación. Universidad de Granada
}

C/ Periodista Daniel Saucedo Aranda, s/n. E-18071 Granada

carcamb1@doctor.upv.es, ssendra@ugr.es, jojiher@dcom.upv.es, jlloret@dcom.upv.es

\begin{abstract}
Resumen- Las nuevas tecnologías son la clave para mejorar la sostenibilidad en el sector agrícola y producir alimentos de calidad. Además, el uso de nuevas técnicas de cultivo, como la hidroponía, basada en la producción de alimentos en agua sin necesidad de cultivarlos en tierra, hace que se puedan analizar mejor las necesidades de la planta $y$ proporcionarle las mejores condiciones de crecimiento. En este artículo presentamos el desarrollo de una red de sensores móviles orientada a monitorizar los patrones de necesidades de las plantas y tomar decisiones inteligentes según la captación de datos ambientales obtenida. La red está compuesta por nodos sensores comunicados con transceptores de radio distribuidos en una red mallada, que podría ser fácilmente adaptada a cualquier tipo de uso a petición del profesional. Esta red ha sido probada en un entorno de agricultura hidropónica. Finalmente el artículo muestra los resultados obtenidos en cuanto a tráfico generado, lo que nos permitirá en un futuro, hacer la red escalable.
\end{abstract}

Palabras Clave- Smart farming, invernaderos inteligentes, red de sensores en horticultura, agricultura de precisión, sensores hidropónicos.

\section{INTRODUCCIÓN}

La incorporación de la tecnología al sector agroalimentario está tomando un papel muy importante en la competitividad de los productores agrícolas. Hoy en día, el mercado demanda productos de mayor calidad y buena presentación por lo que los sistemas de producción se renuevan constantemente.
La cosecha de cultivos en invernadero requiere una captación de datos en diferentes puntos, debido a la naturaleza espacial distribuida, donde las condiciones climáticas pueden variar dependiendo del punto de localización.

El uso de redes de sensores inalámbricas (del inglés, Wireless Sensor Networks, WSN) se está extendiendo de forma exponencial en el sector agrícola [1], ya que se basan en dispositivos inalámbricos, de bajo consumo energético y económico, necesarios en entornos donde se tienen distancias muy extensas y sin posibilidad de acceder a la red eléctrica para suplir al sistema.

Para desarrollar una red de monitorización en instalaciones agrícolas, como por ejemplo, tipo invernadero, podemos decidir entre emplear una infraestructura de comunicaciones inalámbricas, donde la cualificación técnica del personal encargado de la implementación de los dispositivos y su mantenimiento es elevada [2]. No obstante, podemos decantarnos por el uso de redes sin infraestructura que permita la movilidad de dispositivos sin perjudicar las comunicaciones entre ellos y de fácil instalación.

En nuestra propuesta se ha optado por el uso de una tecnología de bajo coste que permite multiplicar los nodos en un sistema, o simplemente desecharlos ante cualquier anomalía de funcionamiento. Se ha optado por el use de los módulos Nordic RF24 que operan en la banda libre en Europa de 2,4 GHz. El bajo coste económico en la tecnología seleccionada de Nordic, nos 
permite aumentar el número de nodos con sensores. Esto supone un aumento de la cantidad de información recogida y la obtención de una mayor cantidad de resultados a partir de los cuales se pueden tomar esas decisiones inteligentes que precisamos para gestionar los entornos monitorizados. Un ejemplo claro de las mejoras de esta es que hace 5 años, únicamente se usaban un conjunto de sensores unitarios de varios tipos (de costes económicos elevados) para todo el invernadero [4], obteniendo un solo dato cada variable (temperatura, humedad, radiación solar, etc.) y se generalizaba para toda la infraestructura cultivable. Con el paso de los años, la tecnología se ha abaratado y han surgido nuevos protocolos de comunicación más eficientes.

Por último, esta información hay que procesarla y mostrarla para darle difusión entre las redes sociales de profesionales. Este es uno de los principales motivos por los que los desarrollos con sensores de este trabajo tengan como finalidad, el envío de datos e integración con la plataforma multimedia PLATEM PA (Plataforma Tecnologica Multimedia en agricultura de precisión) [5], diseñada para crear una interfaz de usuario con reglas inteligentes de decisión en la que pueden interactuar diferentes profesionales del sector agrícola con la finalidad de intercambiar opiniones y resolver problemas con una inmediata actuación en remoto.

PLATEM PA permite la interacción usuario sistema inteligente (riego, invernadero, actuadores, etc.) y facilita el manejo de los cultivos gracias a su motor y reglas de inteligencia, por otro lado se genera un feedback donde usuarios de la plataforma intercambian opiniones y experiencias.

En este artículo se presenta una red de sensores de bajo coste energético para el uso en el sector de la agricultura hidropónica. El sistema se basa en una red de sensores que monitoriza de forma continua un invernadero. Los datos ambientales, calidad de agua y estado de la planta se almacenan en un servidor (la nueve)donde los agricultores y profesionales del sector pueden ver y usar. Al mismo tiempo, también son usados por un nodo coordinador para controlar los diferentes actuadores como dosificadores de abono, luces, válvulas de agua, etc., que actuarán sobre los propios cultivos. La interfaz gráfica y de control que nos permite acceder a estos datos es la propia plataforma PLATEM PA. La red se ha probado en términos de tráfico generado por los nodos en diferentes condiciones.

El resto del trabajo queda estructurado del siguiente modo. En la Sección II se presentan los trabajos realizados en materia de redes de sensores y tecnología de comunicaciones inalámbricas en varios sectores, especialmente en agricultura. La sección III describe el problema del control del clima del invernadero en cultivos hidropónicos. Posteriormente, se analiza el desarrollo de un sistema inteligente de control basado en eventos y la WSN para el control de fertirrigación y condiciones ambientales correctas en relación a temperatura de efecto invernadero, calidad del agua y nutrientes y radiación solar. Los dispositivos usados y desarrollo del frameworks de comunicación de nodos son descritos en la sección IV. En la sección V se detalla los resultados de los test del sistema. Finalmente, las conclusiones y trabajos futuros se exponen en la sección VI.

\section{TRABAJOS RELACIONADOS}

Las redes de sensores se caracterizan por su fácil adaptación a diferentes ámbitos de trabajo, como los sectores de la industria, agricultura, entornos forestales, marinos, militares, etc. Pero existen sectores donde este tipo de sistemas de sensores deben ser instalados en áreas donde no existe corriente eléctrica para alimentarlos. En este caso surge la necesidad de proponer nuevas posibilidades en el diseño de dispositivos capaces de realizar trasmisiones de datos más polivalentes en este medio y con menor consumo energético. Como nos muestra S. Sendra et al. [6] se ha realizado un estudio de red de sensores dedicada a la detección y verificación de incendios en los bosques. Esta red se caracteriza por el uso de cámaras IP creando una red de monitorización conectada entre sí, que es capaz de orientar las cámaras hacia el punto de detección de anomalías mediante sensores con el objetivo de verificar la presencia del incendio.

C. Cambra et al. [7] desarrolló una red de comunicaciones bajo el protocolo 800.11ab destinada a la detección de alpinistas en zonas rurales y alta montaña que pudieran necesitar algún tipo de ayuda. La propuesta se basa en un sistema de aeronave no tripulado capaz de crear una red de comunicaciones en zonas de alta montaña dedicada a la búsqueda y control de senderistas y montañeros.

Existen trabajos más focalizados en el campo de la agricultura de precisión, donde se desarrollaron redes de sensores y tele-gestión de programadores de riego. Este es el caso del trabajo presentado por C. Cambra et al. [5] donde podemos ver una red de comunicaciones inalámbricas de bajo consumo basada en el uso de transmisores de radio en la banda ISM de $868 \mathrm{MHz}$ para crear un sistema inteligente de riego a través de comunicaciones, programadores y sensores de bajo consumo energético en agricultura extensiva al aire libre.

Existen otros proyectos de monitorización de variables agronómicas en invernaderos a través de redes de sensores y comparativas de diferentes tecnologías dentro de las redes 802.15.4 que han tenido buena aceptación como la presentada por H. Ibayashi [8] donde expone el uso de redes funcionando a una frecuencia de $429 \mathrm{MHz}$ y a $2,4 \mathrm{GHz}$ en Japón mostrando menores tasas de error el uso de frecuencias en $429 \mathrm{MHz}$ o Sensorscope en el que G. Barrenetxea nos presenta un sistema de monitorización ambiental usando nodos de comunicación en la banda $868 \mathrm{MHz}$ [9], son proyectos que emplean los estándares IEEE 802.15.4, para la creación de WSN. 
Tabla I

PROTOCOLOS DE COMUNICACIONES INALAMBRICAS

\begin{tabular}{cccc}
\hline \hline Protocolo & RF24 & WiFi 802.11 & LoRa WAN \\
\hline Frecuencia & $2.4 \mathrm{GHz}$ & $2.4 \mathrm{GHz}$ & $868 / 433 \mathrm{MHz}$ \\
Tamño Paq. & $32 \mathrm{Bytes}$ & $1480 \mathrm{Bytes}$ & $256 \mathrm{Bytes}$ \\
Cobertura & $50 \mathrm{~m}-1 \mathrm{~km}$ & $200 \mathrm{~m}-10 \mathrm{~km}$ & $1 \mathrm{~km}-20 \mathrm{~km}$ \\
Consumo & $20 \mathrm{uA} / 8 \mathrm{~mA} /$ & $10 \mathrm{uA} / 60 \mathrm{~mA} /$ & $2,8 \mathrm{~mA} / 38,9 \mathrm{~mA} /$ \\
Idle/ Rx $/ T x$ & $8,4 \mathrm{~mA}$ & $215 \mathrm{~mA}$ & $50 \mathrm{~mA}$ \\
Ancho de & $256 \mathrm{Kbps} /$ & $150 / 300 \mathrm{Mbps}$ & $250 \mathrm{bps} / 5 \mathrm{Kbps}$ \\
Banda & $2 \mathrm{Mbps}$ & & \\
Precio & $0,75 \$$ & $7 \$$ & $15 \$$ \\
\hline \hline
\end{tabular}

Como muestran las diferentes tecnologías inalámbricas (Ver Tabla I), RF24 es la unica que nos oferce una solución compacta con muy poco consumo energético y con un coste claramente inferior a los demás, se observa una diferencia entre los consumos energéticos del uso de un transceptor bajo WiFi al uso de un RF24, con el que podemos asegurar las comunicaciones durante un tiempo de vida de batería bastante extenso. No es tanta la diferencia entre RF24 y un transceptor LoRa, pero si podemos observar que el coste económico de un dispositivo LoRa es superior al coste de RF24, donde en muchos proyectos con un gran número de nodos, puede suponer un incremento inasumible. La tecnología RF24 es la que mejor se adapta al planteamiento inicial y por ello las comparativas realizadas con tecnologías estudiadas en anteriores trabajos, muestran un gran potencial de la red RF24.

El trabajo presentado se centra en el uso del módulo RF basado en el chip Nordic nRF24L01, es ultra compacto y de muy bajo consumo. Trabaja en la banda ISM a una frecuencias de $2.4 \mathrm{GHz}$ (frecuencia libre) con modulación GFSK (Modulación por desplazamiento de frecuencia Gausiana) y es ideal para proyectos de telemetría, control de periféricos, industria y afines. Incorpora un transceptor RF de $2.4 \mathrm{GHz}$, un sintetizador $\mathrm{RF}$, algoritmos de control de errores y un acelerador para trabajar con interfaz SPI. En un futuro se verá el avance de LoRa y la disminución de costes de sus módulos, pero actualmente Nordic RF24 puede ser una alternativa muy buena y barata en redes de sensores agrícolas, demandadas por los profesionales del campo.

\section{SiSTEMA INTELIGENTE DE MANEJO DE CULTIVOS HIDROPÓNICOS}

Esta sección expone los problemas actuales que los cultivos hidropónicos presentan y muestra cómo nuestra propuesta los puede solucionar. El cultivo hidropónico es un método de cultivar plantas usando agua y minerales en vez de tierra. La principal ventaja es el control total del alimento de la planta y la no necesidad de disposición de superficie cultivable, ya que la hidroponía suele expandirse en vertical.

\section{A. Problema de control en los cultivos hidropónicos}

El crecimiento del cultivo está influenciado principalmente por las variables climáticas ambientales que rodean a los cultivos y por la cantidad de agua y fertilizantes suministrados por el riego. Esta es la razón principal por la que un invernadero es ideal para el cultivo, ya que constituye un entorno cerrado en el que las variables climáticas y fertirrigación pueden ser controladas para permitir un óptimo crecimiento y desarrollo del cultivo [10]. El clima y la fertirrigación son dos sistemas independientes con diferentes problemas de control. A priori, se conocen los requisitos de agua y nutrientes de diferentes especies de cultivos $\mathrm{y}$, de hecho, los primeros sistemas automatizados fueron los que controlaban estas variables estáticas. Una simplificación a través de la automatización inteligente consiste en suponer que las plantas reciban la cantidad de agua y fertilizantes que requieren en cada momento, según los datos que retornan.

\section{B. Nuestra Propuesta}

Aunque el sistema se compone de varias partes, en este trabajo nos centramos en la implementación del sistema de recogida de parámetros, que conlleva la configuración de la red, monitorización de parámetros, algoritmos de decisión y control de dispositivos actuadores. En la Fig. 1 se describe la parte del sistema relacionada a nuestro estudio.

Tanto los actuadores como la trasmisión de datos de los dispositivos con sensores se realizan de manera inalámbrica. En este caso se ha optado por el uso del transceptor NRF24L01 de la compañía Nordic semiconductor [11]. El chip de comunicaciones elegido es un Atmel de 8 bits, que se caracteriza por su bajo consumo y podemos encontrarlo en un formato comercial ya ensamblado sobre una placa electrónica con entradas para sensores, sockets de conexión del transceptor de radio y porta pilas [12].

Podemos encontrar algunas propuestas y recomendaciones de uso del transceptor NRF24L01 pero en todas ellas, únicamente se habla de la parte de la trasmisión de datos desde un nodo a su coordinador. En trabajos anteriores con el transceptor NRF24L01, el patrón del protocolo de comunicaciones se basaba en envíos tx desde el nodo a la red mesh para que llegara finalmente al coordinador, estas redes se desarrollan principalmente en redes de sensores para él envió de variables eventuales. En este trabajo, la red incluye una serie de actuadores móviles que necesitan recibir una serie de parámetros para su correcto funcionamiento. En la implementación de una red mesh se tiene una tabla de enrutamiento en ambos sentidos que puede ralentizar el sistema de comunicaciones. Como novedad en nuestra propuesta de red mesh, se ha diseñado un sistema de envío de mensajes basado en los identificadores de los nodos, en lugar de emplear en una tabla de enrutamiento prefijada. Este proceso es similar al tipo de envío que se realiza en un broadcasting, con la peculiaridad de generar un buffer o pila sobre el mensaje. Además, para realizar la trasmisión solo es necesario conocer el identificador del destino y se va generando un buffer con los identificadores recorridos con un modo de reintentos, si fuera necesario. 


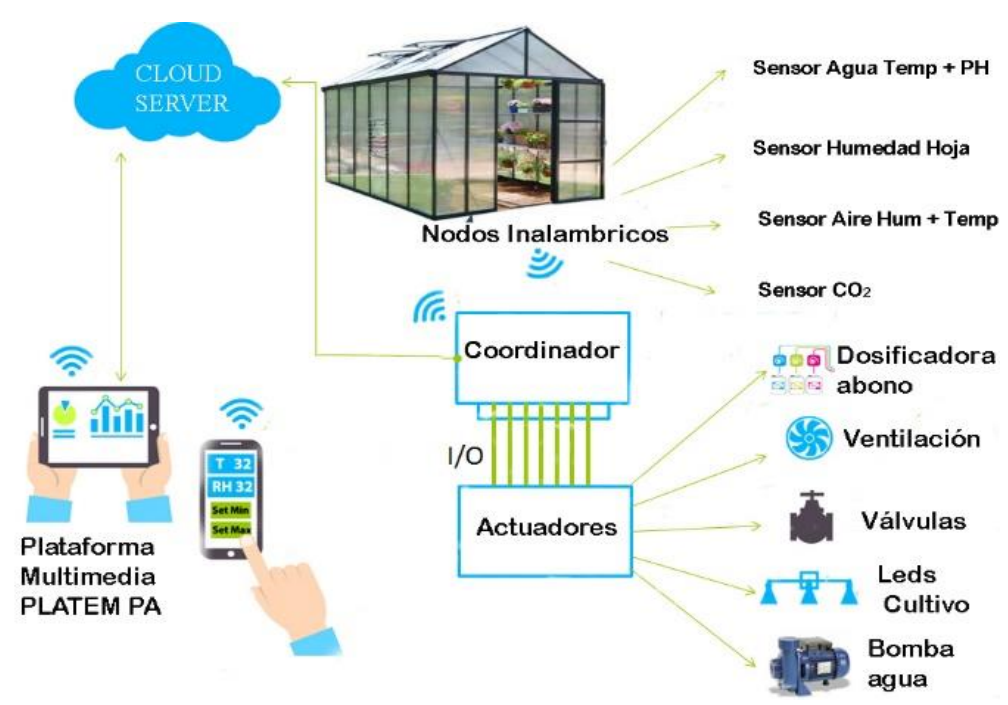

Fig. 1. Esquema de dispositivos del Sistema.

Como podemos ver, tanto la comunicación con las principales ventajas de esta configuración son las siguientes:

- Los nodos no necesitan tablas de enrutamiento para definir el camino más corto.

- Cada nodo usa una configuración de retardo (algoritmo de retardo según identificador) al envío de los mensajes, para evitar colisiones.

- Se pueden insertar nodos nuevos en la red sin la necesidad de modificar ni añadir parámetros identificativos a una tabla de encaminamiento.

- Los nodos se pueden mover, sin perder comunicación, a nivel de una tabla de enrutamiento, supone generar una nueva ruta más óptima.

- No requiere de un nodo central para la gestión de las tablas de enrutamiento.

Creemos que esta propuesta supone un gran avance para el sector, ya que las redes de sensores y en nuestro caso la descrita en este trabajo, va a poder ser usada por agricultores y profesionales del campo que quiere tecnificar sus sistemas de cultivo, pero no desean costear soporte técnico cualificado. Además la inclusión de un nuevo modo no implicará que tengan que modificar parámetros en el sistema hidropónico, es decir, nos encontramos frente a un sistema sencillo y de fácil adaptación al entorno de trabajo.

\section{IV.ARQUITECTURA DEL SISTEMA}

En esta sección se describe los diferentes dispositivos usados como nodos con sensores, coordinadores de red y actuadores. A nivel de comunicaciones se ha implementado un framework basado en comunicaciones sin infraestructura con encaminamiento multisalto lo que permite una movilidad de nodos dentro de la red mallada WSN, existen diferentes trabajos relacionados con las arquitecturas de protocolo [13] que nos proporcionan la base de las funciones de encaminamiento.

\section{A. Dispositivos}

Los dispositivos usados en la red son nodos inalámbricos, con un consumo de energía ultra bajo, capaces de transmitir la información de los sensores conectados. La Fig. 2 muestra nos nodos inalámbricos empleados. Una de los motivos por los que se ha elegido este dispositivo, además de por su bajo consumo, es su pequeño tamaño (Dimensiones 30,5 x $67,0 \mathrm{~mm}$ ). Los sensores están conectados a los nodos, de forma muy sencilla a través de los sockets, dedicados a la toma de diferentes datos necesarios para la toma de decisiones de funcionamiento del sistema de cultivo y análisis del crecimiento del cultivo. Por un lado, como muestra la Fig. 3 incorporamos sensores para el control de los sistemas de producción para comprobar su correcto funcionamiento, como puedan ser nivel de flujo de agua, nivel de caudal o detección de agua en un punto. Otro tipo de sensores integrados en el sistema son los dedicados a la medición de parámetros de calidad y niveles de elementos nutricionales de las plantas, los encargados de determinar las actuaciones de aporte nutricional, luz, aire, temperatura, etc. En la Fig. 4 se muestra algunos ejemplos de sensores usados como humedad de la hoja, nivel de $\mathrm{pH}$ y temperatura del agua.

En cuanto al nodo base (Ver Fig. 5), este se considera el punto central dedicado a recoger la información de la red de sensores y comandar operaciones a los actuadores añadidos al sistema de cultivo, este a su vez está conectado a internet a través de la red móvil con tecnología $3 \mathrm{G}$ y mantiene comunicación bidireccional con la Plataforma multimedia PLATEM PA, que es la encargada de almacenar todos los datos, crear patrones de notificaciones o eventos y tomar imágenes, gracias a una pequeña cámara.

El protocolo de comunicaciones seleccionado está orientado al uso de la red de radio en la frecuencia de $2,4 \mathrm{GHz}$ en modo mallado o Mesh donde los identificadores de los nodos son identificadores únicos y es una red dinámica ya que es posible que los 
dispositivos entren y salgan de la red. Debido a esta estructura, es sencillo para cualquier nodo comunicarse con el nodo maestro.

Una cosa a tener en cuenta es la naturaleza dinámica del protocolo RF24Mesh, y la necesidad de verificar la conectividad a la red. Para los nodos que están transmitiendo constantemente es conveniente comprobar la conexión y/o renovar la dirección cuando la conectividad falla cada pocos segundos. Los nodos que no están transmitiendo activamente, deben configurarse para probar su conexión a intervalos predefinidos, para permitir que se vuelvan a conectar según sea necesario. En el caso de nodos en modo Sleep sólo estarán en línea temporalmente, por tanto, es adecuado liberar la dirección antes de desconectarse y solicitar una dirección al despertar.

Este protocolo es capaz de transmitir cargas útiles sin que la red devuelva una respuesta. Si utiliza únicamente este método de transmisión, el nodo también debe configurarse para verificar periódicamente su conexión a través de la instrucción mesh.checkConnection (). La comunicación de nodo a nodo requiere que las consultas de dirección se envíen al nodo maestro, ya que los nodos individuales pueden cambiar de dirección en cualquier momento.

El motivo por el que nos hemos declinado por el uso de este dispositivo se centra en varias ventajas que a priori hemos visto que se adaptan bien a sistemas de sensores. Por una parte, el NRF24L01 es muy barato (hasta 10 veces) aunque por el contrario, el tamaño de mensaje es más pequeño, siendo de 32 Bytes, es decir, si la cabecera del protocolo es grande, el espacio para la trama de datos es pequeño [14]. El protocolo utiliza sólo 8 Bytes cuando se rellenan todas las condiciones, redundancia, ACKs, checksum y repeticiones.

El campo de dirección es muy similar al protocolo IPV4, pero en nuestro caso, usamos valores hexadecimales con un tamaño de 5 bytes. Otra peculiaridad es el uso de tuberías de comunicación, tiene como máximo 5 flujos por lo que cada direccionamiento en uso se asigna a una de ellas. El Pid (Packet ID) que se ha empaquetado se particiona en el tipo de mensaje (por ejemplo, la temperatura, la humedad,...), el valor y si es una difusión o una solicitud punto a punto al nodo final de solicitud o respuesta. La parte del origen y el destino del mensaje viene asignado por las direcciones y el uso de las tuberías de comunicaciones. El CRC se calcula sólo cuando se alcanza el nodo final. Si no es así, se activa el reintento. Las marcas de las repeticiones son más eficientes y más rápidas para reducir el tiempo de trabajo de la red.

\section{RESUlTADOS}

Esta sección muestra los resultados de rendimiento, por un lado a nivel de datos erróneos en los envios de los sensores y por otro en cuanto a tráfico generado para 2 escenarios de transmisión diferentes.

Como se observa en la Fig. 6, es posible que por problemas del ambiente o defectos en componentes físicos del sensor, se puede dar el caso que los datos sean erronos y estén fuera de un umbral de trabajo. Por ello mostramos una tasa de error en un sensor usado, mostrando una tasa de error cada 60/70 minutos de capturas realizadas cada minuto $(1,6 \%)$.

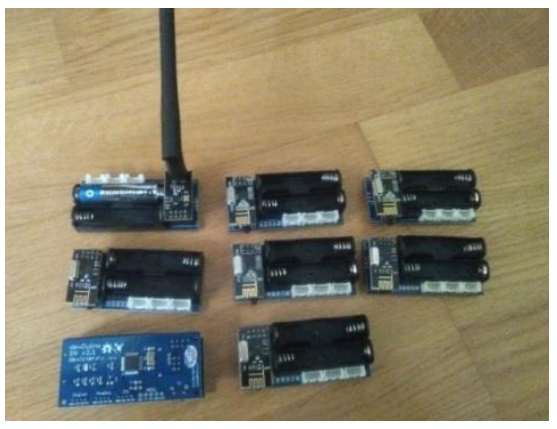

Fig. 2. Imagen de nodos del sistema

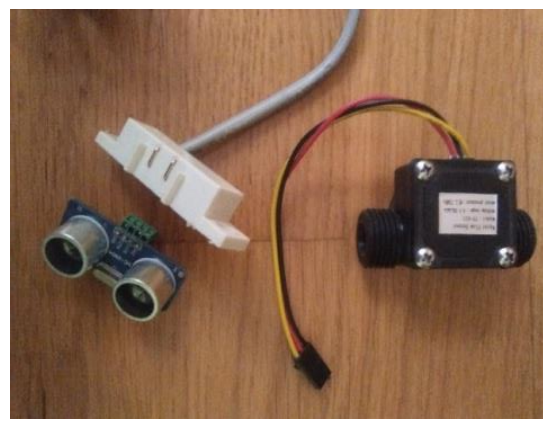

Fig. 3 Sensores del funcionamiento

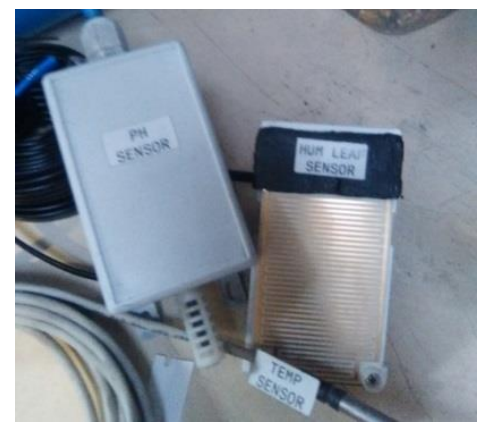

Fig. 4. Sensores de estado del agua y cultivo sistema.

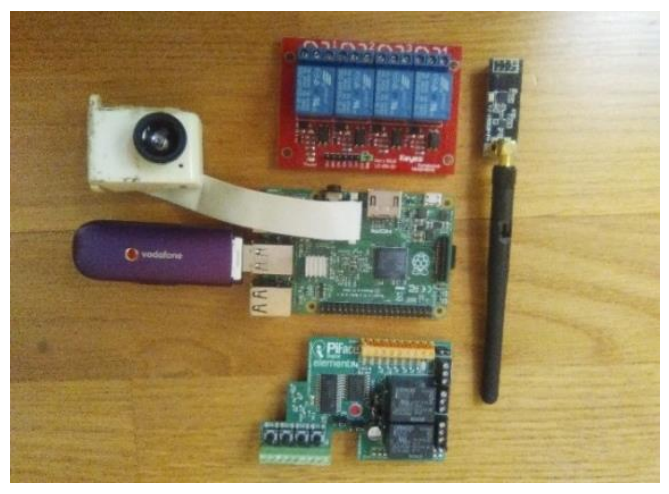

Fig. 5. Nodo central con actuadores, conectividad radio y $3 \mathrm{G}$.

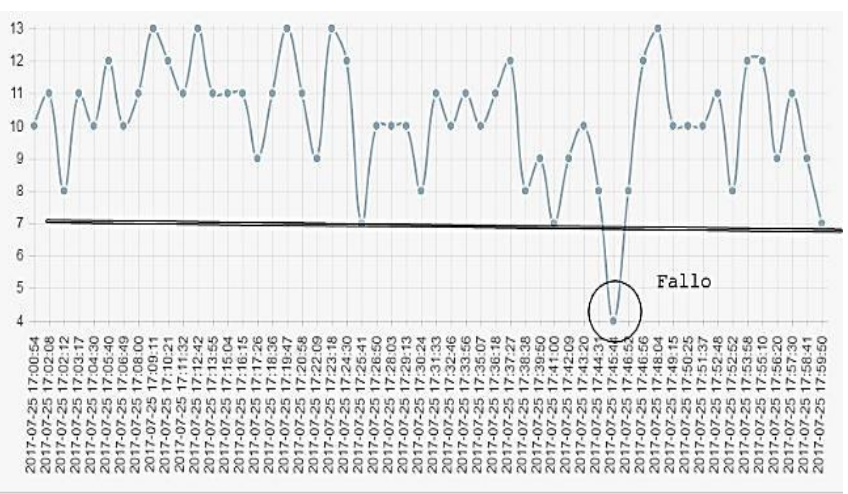

Fig. 6. Detección de datos erroneos, sensor flujo 


\section{A. Escenarios de pruebas de la red de comunicaciones}

El sistema de radio soporta una función de confirmación automática (autoACK), activada de forma predeterminada, en la que la radio receptora cambia a modo de transmisión después de la recepción y reconoce la recepción de datos. Esto supone mantener un canal bidireccional.

En el primer test se crea un escenario sencillo con 3 nodos, A, B y C, con el siguiente intercambio de mensajes:

1. A envía a $B$

2. A envía a $\mathrm{C}$

3. B responde $\mathrm{A}$

4. A ya sabe que B tiene el mensaje correcto.

5. $\mathrm{C}$ responde $\mathrm{A}$

6. A ya sabe que $\mathrm{C}$ tiene el mensaje correcto.

La Fig. 7 muestra el consumo en bytes de la red para el intercambio de mensajes explicado. Acorde con las recomendaciones de los fabricantes, si mantenemos la confirmación automática activa, estamos limitados a

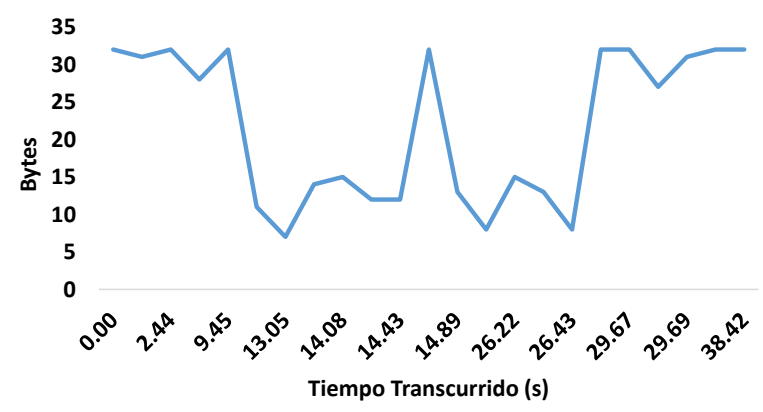

Fig. 7. Tráfico generado en la conexión punto a punto.

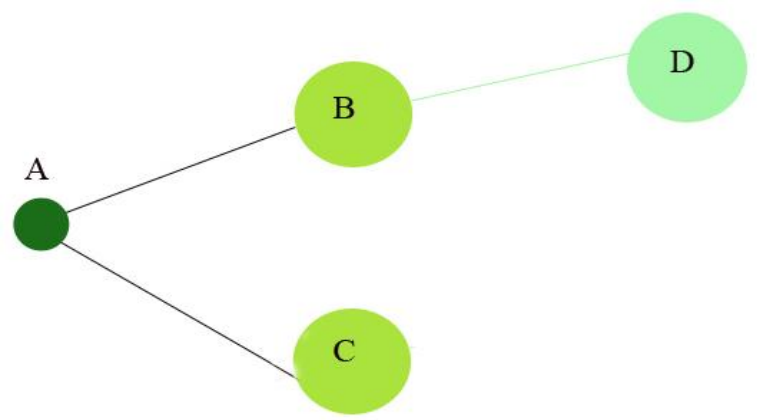

Fig. 9. Árbol de nodos en pruebas.

En la Fig. 9 se muestra el árbol de nodos de las pruebas con red mallada donde B depende de A y da comunicación a D. Como podemos observar en la Fig. 10, existen ocasiones en el que los paquetes no alcanzan su destino, de ahí que tengamos algún CRC error (longitud 10/12 bytes) y se reintente el envío.

El modo de difusión elegido puede tener problemas de colisión, aun con el algoritmo de desfase introducido. Esta tarea será un futuro análisis [17] a estudiar en siguientes trabajos. tener solamente 5 nodos en la red. Por lo que para este trabajo será necesario desactivar esta función.

La siguiente prueba de envío de datos se realiza con la función Auto-ACK desactivado. Se emplean en este caso, 4 nodos, A, B, C y D en forma de red mallada. El intercambio de mensajes que se realiza es el siguiente:

1. A envía

2. B, C, D escuchan

3. B tiene el mensaje con el ID correcto.

Como nos muestras la Fig. 8, se ha realizado un envío de mensajes sin auto respuesta ACK y se puede ver alguna pérdida de trasmisiones, posiblemente por colisión de los paquetes. A la hora de implementar una red mallada comprobaremos la recepción de mensajes con el ACK de respuesta del destinatario.

Por último, realizaremos una prueba con 4 nodos con Auto-ACK activo donde cada radio está vinculado a una dirección [15] (Bit Masks) y tubería que utiliza para comunicar con su padre o hijo. El nodo, una vez que escucha el paquete puede aceptarlo [16], encaminarlo a su padre o encaminarlo a su hijo: A flujo 1, B flujo 2, C flujo 3 y D flujo 4.

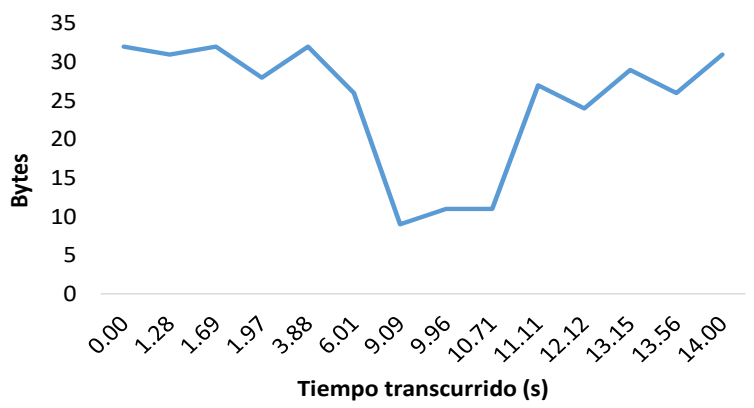

Fig. 8. Tráfico generado en las pruebas de red mallada.

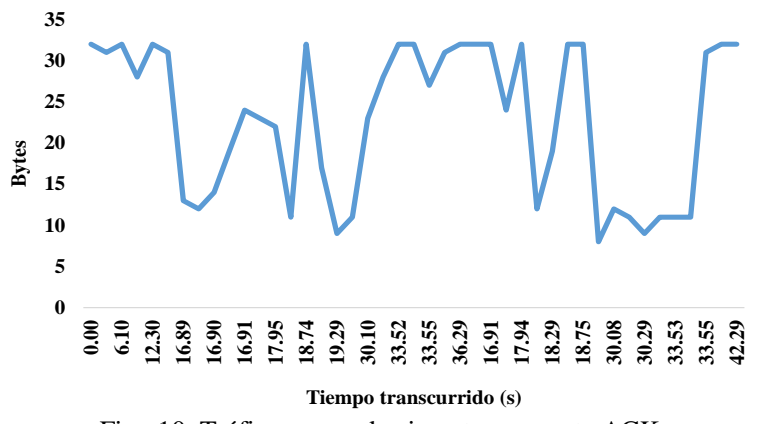

Fig. 10. Tráfico generado sin auto respuesta ACK.

\section{B. Control de bajo consumo energético}

Para maximizar el tiempo de funcionamiento de una batería cuando se emplea el módulo NRF24L01, debemos configurar una serie de mecanismos de ahorro de energía. Las líneas de código implementadas que nos permiten obtener este ahorro de energía se muestran en la Fig. 11. Para asegurar un ciclo de vida de batería más largo, se puede reducir la frecuencia del microcontrolador a $1 \mathrm{MHz}$ y bajar el voltaje de funcionamiento. Podríamos elegir trabajar a $1.8 \mathrm{~V}$ en vez de $3.3 \mathrm{~V}$ 


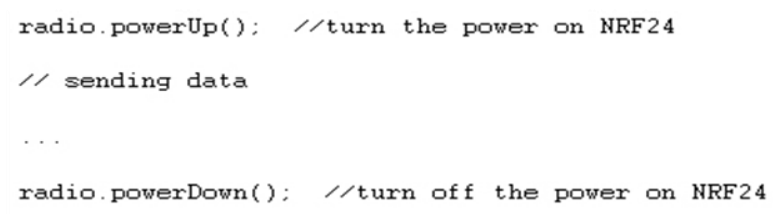

Fig. 11. Encendido y apagado del módulo de radio.

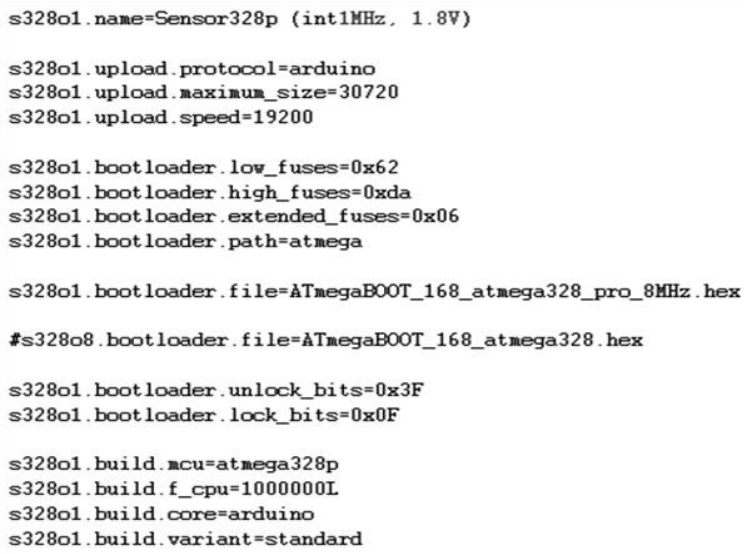

Fig. 12. Código para reducción de ciclos de reloj.

La Fig. 12 muestra el código perteneciente a la disminución de frecuencia de trabajo del chip, para descender el consumo energético. Para pasar el nodo a modo dormido será necesario implementar una función en la cual comparamos varias casuísticas que dependerán de los tipos de sensores conectados, estas pueden ser:

- $\quad$ Si el módulo es activado por una interrupción de una entrada (nivel de agua en un deposito)

- $\quad$ Si se despierta cada un tiempo definido de captura de datos (temp, humedad,..)

- $\mathrm{Si}$ se despierta por un tiempo para hacer comprobaciones de red (pulling) por si tiene alguna recepción pendiente.

En nuestro caso, se ha definido un tiempo de recepción cada dos segundos y de captura de datos cada 15 segundos. En el caso de pulling es necesario despertar el nodo y el radio cada dos segundos y mantenerlo despierto $500 \mathrm{~ms}$ ya que el trasmisor necesita dos segundos de preámbulo en cada paquete. La función implementada se muestra en la Fig. 13.

Existes diferentes enfoques en el modo de trabajo de este tipo de dispositivos y el modo de dormirlos y despertarlos [18], pero en definitiva, estos será elegidos en función de las condiciones de uso de los sensores. En cualquier caso, siempre nos permitirá alargar la vida útil de las baterías o capacitadores de almacenamiento.

Finalmente, la Fig. 14 muestra los valores de consumo de corriente de estos nodos, cuando están funcionando a $3.3 \mathrm{~V}$. Cuanddo el nodo está transmitiendo datos los valores de corriente se sitúan en torno a los $30 \mathrm{~mA}$. Cuando el nodo está transmitiendo datos, su consumo se incrementa hasta los $49 \mathrm{~mA}$. Finalmente, en relación al consumo de energía cuando el nodo se encuentra en el modo sleep, el consumo de corriente se reduce radicalmente hasta los $90 \mu \mathrm{A}$.

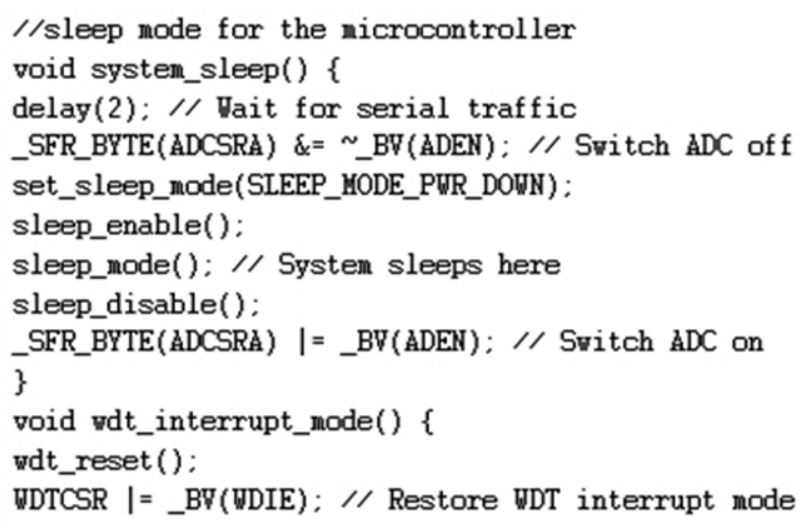

Fig. 13. Código de control de estados del nodo e interrupciones

En este tipo de sistemas, existe la posibilidad de usar placas solares para una alimentación extra, con el inconveniente de la movilidad y orientación forzada hacia la luz solar. En estos casos, se debe hacer un balance entre la energía consumida para el funcionamiento del nodo y el movimiento de la placa solar y la energía solar que es capaz de captar la placa usada.

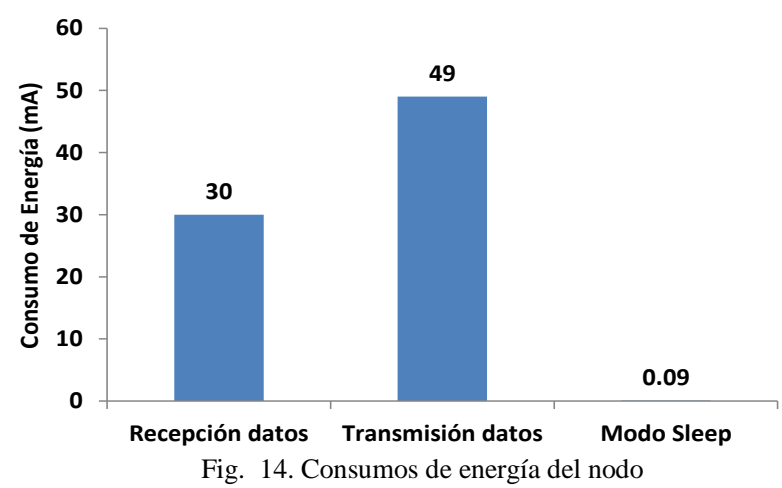

\section{CONCLUSIONES}

La evolución de la sociedad tiende a proponer nuevos retos alimentarios relacionados con la evolución de nuevos sistemas de cultivo, como es el caso de los hidropónicos que está fuertemente ligada a las redes de sensores [19]. Las WSN son sin duda la mejor solución para el seguimiento cultivo y su correcta producción.

Se está tendiendo al uso de tecnologías ecológicas energéticamente eficientes a través de los algoritmos y patrones de uso dedicados dentro de la Plataforma multimedia específicas. Este es el caso de PLATEM PA.

En este trabajo se ha presentado una WSN de bajo consumo que implementa un sistema autónomo construido con el objetivo de automatizar el proceso 
productivo en el cultivo intensivo [20]. El diseño de la red de sensores inalámbricos se centra en el uso de nuevos diseños de redes malladas caracterizadas por alcanzar un consumo realmente bajo y la independencia de los nodos con el mínimo soporte técnico posible.

Dentro de este sistema la captación de parámetros ambientales, datos del cultivo y calidad de agua y nutrientes son cuantificados en pocos segundos gracias a la red de sensores desplegada en el invernadero, la corrección de parámetros puede ser llevada a cabo en un corto espacio de tiempo y reaccionar con antelación a posibles problemas o casuísticas distintas. Hemos observado que los nodos de sensores en algún momento emiten datos erróneos asíncronamente Aprovechando el gran número de nodos de sensores desplegados, es necesario tener una regla de control bajo un umbral de variables asignadas, para filtrar datos totalmente alejados de parámetros reales del sistema. Si un nodo es problemático continuamente, se detecta y se desecha esa información.

La sociedad demanda cada vez más cultivos más saludables y que puedan ser transformados con el menor impacto ambiental posible [21], de ahí que en pocos años veamos un despliegue exponencial de huertos urbanos altamente tecnológicos. Como futuros desarrollos y avances en este tipo de redes de sensores de bajo coste, deseamos continuar con el análisis y depuración del protocolo de comunicaciones en redes malladas con esta tecnología, para solventar el problema leve de pérdida de paquetes, que suponen hacer mayores reintentos de envío del mensaje y conlleva a un mayor consumo de energía. Una vez estabilizado ese problema, las líneas de trabajo se orientarán a crear mayor inteligencia a la plataforma tecnológica, tanto para la difusión de mensajes entre los responsables de los sistemas, como en actuaciones automáticas o acciones para recortar los periodos de anomalías en los sistemas de producción. Compartir los datos de la plataforma PLATEM PA con robots encargados de tareas de supervisión y de procesos mecanizados será clave en la integración de sistemas $100 \%$ autónomos sin tener el factor humano físicamente en el lugar de operaciones y pueda hacerlo en remoto.

\section{REFERENCIAS}

[1] D.D. chaudhary, S.P Nayse, L.M. Waghmare, "Application of Wireless Sensor Network for Greenhouses Parameter Control in Presicion Agriculture", International Journal of Wireless \& Mobile Networks, 2011, vol 3.No 1, Pp. 140-149.

[2] S.R. Boselin, M. Pradeep, E. Gajendran, "Monitonig Climatic Conditions Using Wireless Sensor Networks", A multidisciplinary Journalof Scientific Research \& Education, 2017, Vol. 3, No. 1, Pp.179-184.

[3] S Sendra, J Lloret, C Turro, JM Aguiar, IEEE $802.11 \mathrm{a} / \mathrm{b} / \mathrm{g} / \mathrm{n}$ short-scale indoor wireless sensor placement, International Journal of Ad Hoc and Ubiquitous Computing, 2014, Vol.15, No. $1-3$, pp. $68-82$

[4] S. Janos, G, Martinovic, I. Matijevics, "WSN Implementation in the Greenhouses Environment Using Mobile Measuring Station", International Journal of Electrical and Computer Engeenering Systems. 2010, Vol 1, No. 1, Pp. 37-44.
[5] C. Cambra, S. Sendra, J. Lloret, L. Garcia, "An IoT ServiceOriented System for Agriculture Monitoring, In proceedings of the Intenational Conference on Communications 2017, May 2125, Paris (Francia).

[6] A. Tozounis, N. Katsoulas, K.P Ferentinos T. Bartzanas, C Kattas, "Development of a WSN for Greenhouse Microclimate Distribution Monitoring", University of Targoviste-Agriculture, 2016, Vol.10, No. 1, Pp. 7-13.

[7] J. Lloret, M. Garcia, D. Bri, S. Sendra, "A Wireless Sensor Network Deployment for Rural and Forest Fire Detection and Verification”, Sensors, 2009, Vol. 9, No. 11, Pp. 8722-8747.

[8] C Cambra, S Sendra, J Lloret, L Parra. "Ad hoc network for emergency rescue system based on unmanned aerial vehicles", Network Protocols and Algorithms, 2016, Vol 7, No 4, Pp. 7289.

[9] H. Ibayashi, Y. Kaneda, J. Imahara, N. Oishi, M. Kuroda and H. Mineno, A Reliable Wireless Control System for Tomato Hydroponics" Sensors 2016, 16, 644 .

[10] G. Barrenetxea, F. Ingelrest, , G. Schaefer, M. Vetterli, "Wireless sensor networks for environmental monitoring: The sensor scope experience", In proceedings of the 2008 IEEE International Zurich Seminar on Communications, March 12-14, 2008, Zurich (Switzerland). (pp. 98-101).

[11] C. Yeng, S. Yuling, W. Zhongyi, "Connectivity of Wireless Sensor Networks for Plant Growth in Greenhouse", International Journal of Agricultural and Biological Engineering of Beijing, 2016, Vol. 9, No 1, Pp. 89-98.

[12] Nordic Semiconductor, RF specialist in ultra low power wireless communications. Disponible en http://www.nordicsemi.com/eng/Products/2.4GHzRF/nRF24L01 , [Último Acceso, 20 de Abril de 2017]

[13] Caracteristicas de Arduino Nrf24L01. Disponible en: http://playground.arduino.cc/InterfacingWithHardware/ Nrf24L01, [Último Acceso, 20 de Abril de 2017]

[14] C. Zhurong, H. Chao, L. Jingsheng, and L. Shoubin, "Protocol architecture for wireless body area network based on nrf24101", In proceedings of the 2008 IEEE International Conference on Automation and Logistics (ICAL 2008), Sept 1-3, 2008, Chindao, (China). (pp. 3050-3054).

[15] V. Gupta, P. Raspaile, "Low Cost Standard Internet of Things", International Journal of Engineering Science \& Advanced Technology, 2015.Vol.5, No.2,pp. 78-80.

[16] Arduino Bit Masks definitions. Disponible en: https://www.arduino.cc/en/Tutorial/BitMasks, Mayo 2017 [Último Acceso, 20 de julio de 2017]

[17] P. Mutukhumaran, R. Paz, R. Spinar, " MeshMAC: Enabling Mesh Networking over IEEE 802.15.4 through Distributed Beacon Scheduling", In proceedings of the International Conference on Ad hoc Netwroks, ADHOCNETS 2009, September 22-25, 2009. Niagara Falls, Ontario,(Canada). (pp. 561-575)

[18] T. Camilo, C. Carretero, J. da Silva, "An Energy-Efficient AntBased Routing Algorithm for Wireless Sensor Networks", In proceedings of the 5th International Workshop on Swarm Intelligence (ANTS 2006), September 4-7, 2006, Brussels, (Belgium). (Pp.49-59).

[19] N. Pantacis, D. Vergados. "Energy efficiency in wireless sensor networks using sleep mode TDMA scheduling", Adhoc Networks, 2009, Vol. 7, No. 2, Pp. 322-343.

[20] L. Ruiz-Garcia, P. Barreir, J.I. Robla, "Performance of ZigBeeBased wireless sensor nodes for real-time monitoring of fruit logistics". Journal of Food Engineering. 2008, Vol. 87, No. 3, Pp. 405-415.

[21] A. Pawloswki, J. Guzman, F. Rodriguez, "Simulation of Greenhouse Climate Monitoring and Control with Wireless Sensor Network and Event-Based Control”, Sensors, 2009, Vol. 9, No. 1, Pp. 232-252.

[22] FAO, Organización de las Naciones Unidas. Disponible en: http://www.fao.org/climate-smart-agriculture/es/, [Último Acceso, 20 de julio de 2017] 